\title{
A Review On Designing Of The Dual Reflector Axially Symmetric Cassegrain Antenna
}

\author{
${ }^{1}$ Divya Gupta, ${ }^{2}$ R. A. Deshpande \\ ${ }^{1,2}$ (Electronics and Telecommunication, K.J.Somaiya College of Engineering, India)
}

\begin{abstract}
Dual reflector antennas are considered as pencil beam antennas that can produce radiation identical to searchlight. Cassegrain Reflector Antenna Design consists of various effects caused by blockage by primary feed or by the subreflector and its effect on overall performance. The objective of the paper is to provide the overview of the designing approach that is used to design the axially symmetric cassegrain antenna. This paper also provides the reader the overview of the various challenges and limitation that are faced by the designer while designing the axially symmetric dual reflector cassegrain system.
\end{abstract}

Keywords: Cassegrain Antenna, dual reflector design, minimum blockage condition, feed system

\section{Introduction}

The parabolic reflector antenna is the most preferred antenna system for many applications this is because of its capability of providing higher gain over a wide bandwidth, availability of accurate modeling techniques and design maturity [4]. One of the essential requirements is to achieve very high cross-polarization discrimination over a specified bandwidth, while maintaining the compactness of the overall antenna system [1]. The cross-polarization refers to the radiation of electromagnetic energy into the polarization other than the desired polarization. It can also be considered as the loss of energy in the unintended direction. The presence of high cross-polarization may result in several undesirable effects. It degrades the overall performance of the system and restricts its use for many applications.

The cross-polarization in a reflector type of antenna system depends on many parameters, e. g., the geometry of the reflector, the focal-length to diameter ratio (F/D) of the antenna system, the reflector surface imperfections, support struts, etc. For example, in microwave radiometers, it reduces the beam efficiency and results in poor spatial resolution [2]-[3]. In case of radar, the high cross-polarization can create boresight-jitter (boresight uncertainty). Boresight uncertainty or boresight fluctuations affect the tracking accuracies and result into unsatisfactory operation of the radar. Axially symmetric dual-reflector antennas (Cassegrain or Gregorian, classical or shaped) are of interest in radio astronomy and in Earth-station antenna technology. The design of such systems is often restricted by factors such as mechanical constraints, the type of feed hom used, and the budget of the project [5].

\section{Geometry}

Consider the geometry of the Cassegrain dual reflector antenna is given in figure1 [5] where $D_{m}$ and $D_{s}$ are the diameter of the main reflector and the sub reflector respectively. $\mathrm{F}$ is the focal distance of the main reflector, $L_{m}$ is the distance between the face center of the feed and the apex of the main reflector, $L_{g}$ is the distance between the apex of the subreflector and the position of the secondary focus. $\theta_{\theta}$ is the angle between the $\mathrm{z}$ axis and the edge ray on the subreflector.

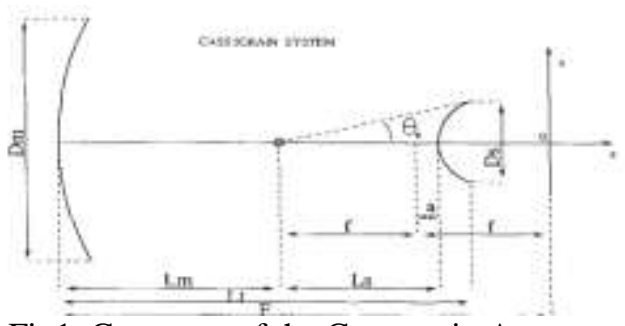

Fig1. Geometry of the Cassegrain Antenna

\section{III.} Few Design Tips

- F/D must be taken in between .25 to .85

- $D_{s} \leq 0.1 \mathrm{Dm}$ for $>>99 \%$ blockage efficiency, as shown in Figure 2[5], and to ensure that the sidelobe levels are not excessive. 


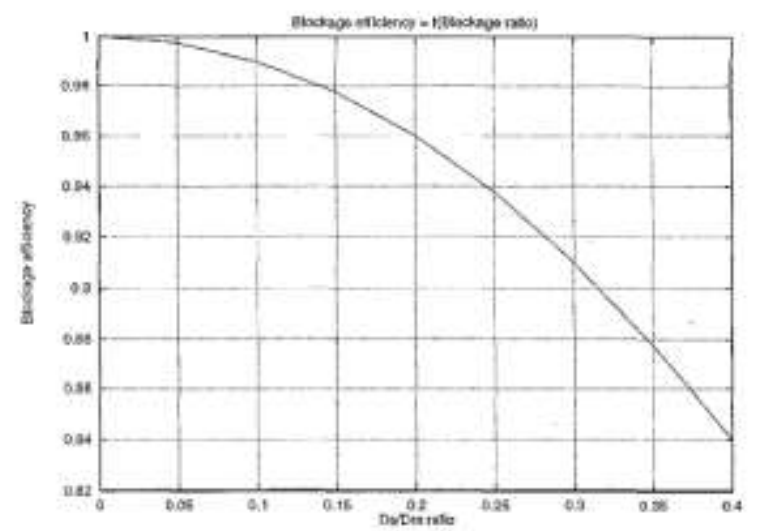

Fig 2. The blockage efficiency as a function of the blockage ratio

- Lm must be chosen keeping in mind the field pattern to be used

- Choose $\theta \mathrm{e}$, to minimize the spillover, i.e., to have an edge illumination on the subreflector of the order of at least -10 to $-15 \mathrm{~dB}$.

\subsection{Minimum Blockage Condition}

If the type of feed to be used is one of the constraints for the antenna design, it is possible to design the antenna to have minimum blockage. In this case, the overall diameter of the feed aperture (including the flange) needs to be known. where Df represents the overall diameter of the feed (fig 2 [5]).

The condition for minimum blockage (the shadow of the subreflector equals to the shadow of the feed as shown in fig 3as given in Hannan[3] as

$$
\frac{F}{2 f}=\frac{D_{s}}{D_{f}}
$$

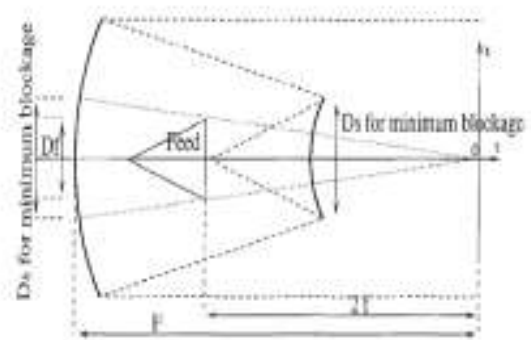

Fig 3. The condition of minimum bloakage by subreflector and feed

\subsection{Design Parameters}

Fig. 1 shows the conventional geometry of axially symmetric Cassegrain antenna. The main reflector is paraboliodal and depends on parameters Dm and F. The subreflector is a convex hyperboloid and depends on parameters Ds, f, and a.

\section{Design Procedure}

The design procedure is based on Milligam[6] and Kildal[7].To design the overall geometry six parameters $D_{m} D_{s}, F, f, a$ and $\theta_{\theta}$.

Two major problems the designer encounters at the first step are two conceptual defections as illumination loss and spillover loss. If the subreflector is assumed as a virtual feed ,the desired main reflector illumination is as Fig.4a,while virtually the main reflector illumination is as Fig.4b.By overlapping these two figures ,the result is as Fig.4c.In this figure two areas are determined separately as the illumination loss and the spillover loss[8].

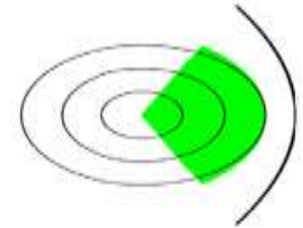

Fig.4a Desired dish Illumination

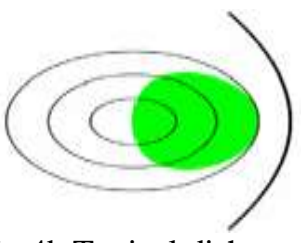

Fig $4 b$ Typical dish illumination

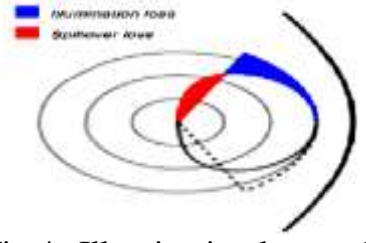

Fig.4c Illumination loss and spillover loss

www.iosrjournals.org 
The starting point is the desired illumination taper between the main reflector and the virtual feed(subreflector).The following procedure is as follows :

\subsection{Diameter of the main paraboloidal dish $(\mathrm{Dm})$}

The main reflector illumination taper in Cassegrain antenna is found to be in between $10 \mathrm{db}$ to $15 \mathrm{db}$ [7]. A plot is shown in fig [5].Since the size of the overall antenna system is restricted by the mechanical constraints and the budget of project, the designer must assigns the first mechanical implementation consideration here on $D_{m}$ according to the prudent antenna size.

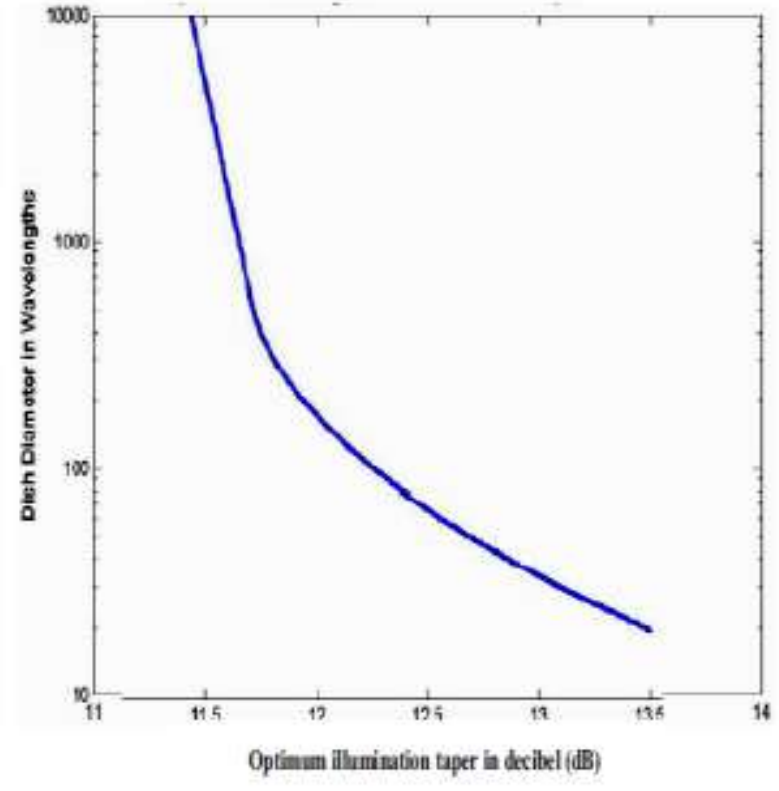

Fig.5 Main reflector diameter Vs. the illumination taper

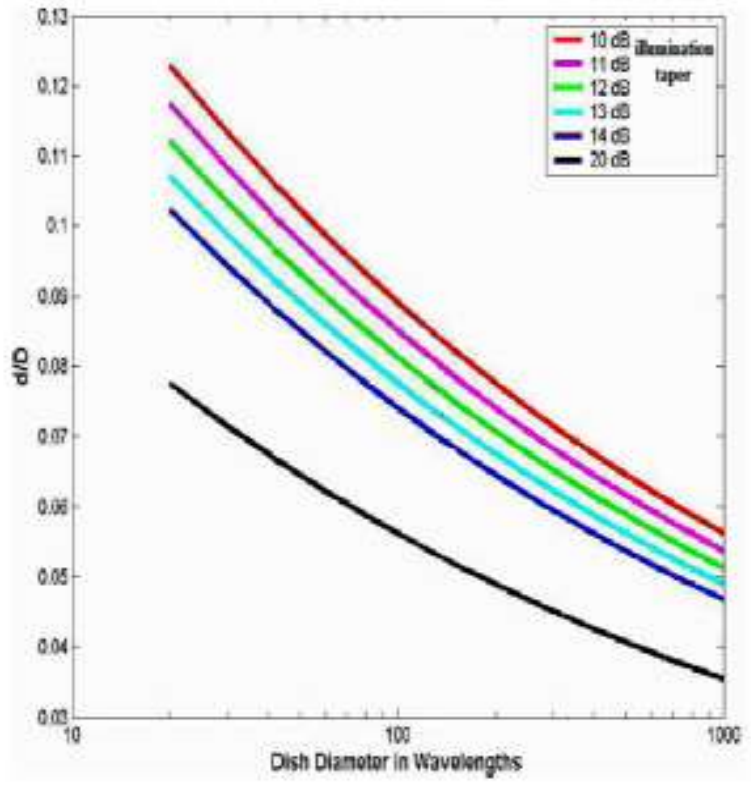

Fig. 6 Ds/Dm vs. main reflector diameter

\subsection{Diameter of the hyperboloid subreflector (Ds)}

In order to minimize the subreflector blockage, a proposed plot is given in[7] showed in fig.(6) This figure give the optimum ratio of the subreflector diameter to the main reflector diameter.

\subsection{Focal distance of the paraboliodal main reflector $(F)$}

The depth of the parabola is given mathematically by equation (2) [9].

$$
x=\frac{D^{2}}{16 F}
$$

Since $\mathrm{F}$ value is inversely proportional to the $\mathrm{Dm}$, appropriate value of the $\mathrm{F}$ should be chosen in order to prevent the main reflector from becoming neither deep nor shallow. Thus the second mechanical implementation consideration is defined on the prudent depth for the main reflector. In general antenna have $\mathrm{F} / \mathrm{Dm}$ ratios between 0.25 and $0.85[5]$

\subsection{Focal distance of hyperboloidal subreflector (2f)}

From [5], $f$ can be easily calculated by equation(3) after knowing the F, $D_{g}$ and $D_{f}$, where $D_{f}$ is the aperture diameter of the feed.

$$
\mathrm{f}=\frac{F D_{f}}{2 D_{s}}
$$

$4.5 \mathrm{Lm}, \theta e, \mathrm{Ls}$ and a

$\mathrm{Lm}, \theta \mathrm{e}, \mathrm{Ls}$ and a are achieved in equation (4),(5),(6) and (7) respectively[5].

$$
\begin{gathered}
L_{m}=F-2 f \\
L_{s}=\frac{2 D_{m f}}{D_{m}-4 F \tan \left(\frac{\theta_{g}}{2}\right]} \\
\theta_{e}=\operatorname{artan}\left[\frac{g F D_{m} D_{s}}{a 2 f F D_{m}-D_{s}\left(16 F^{2}-D_{s}^{2}\right)}\right] \\
a=L_{g}-f
\end{gathered}
$$




\subsection{Eccentricity of the hyperboloid}

The eccentricity $e$, of the hyperboloid is simply given by the equation (8)[5]

$$
e=\frac{f}{a}
$$

\subsection{Feed System Design}

In order to minimize the blockage caused by the feed horn's aperture diameter, the following inequality in Equation (9) must be checked [15].

$$
D_{s}>D_{f} \frac{F}{2 f}
$$

Now the subreflector must be placed in the far-field (Fraunhofer Region) of the feed horn [10], using equation (10).Placing the subreflector in the reactive near field of the horn will cause to an unexpected radiation patter, phase error and undesirable antenna performances. Experiments suggest that the distances close to the half of the Fraunhofer distances are likely acceptable without major problems.

$$
f>2 * \frac{D_{f}^{2}}{a}
$$

\section{Conclusion}

In this paper, a simple overview of the design approach for Cassegrain Antenna is provided by eight prescribed parameters $D_{m}, F, D_{g}, L_{m}, L_{g}, \theta_{g}, f$ and $a . D_{m}, D_{s}$ and $F$ Assigned reasonably to fulfil the mechanical implementation constraints.

\section{References}

[1] Y. T. Lo, and S. W. Lee, Antenna Handbook, Van Nostrand Reinhold Co., New York

[2] J. L. Volakis, Antenna Engineering Handbook, The Mc-Graw Hills Companies, pp. 41.6-41.8.

[3] D. A. Pujara, S. B. Sharma, and S. B. Chakrabarty, "Historical and Planned Uses of Antenna Technology for the Space-borne Microwave Radiometer," IEEE Antennas and Wave Propagation Magazine ,February 2011.

[4] W. V. T. Rusch, "The Current State of the Reflector Antenna Art," IEEE Transactions on Antennas and Propagation, vol. 32, pp. 313-329, April 1984

[5] Christophe Granet, "Designing axially symmetric Cassegrain or Gregorian dual-reflector antennas from combinations of prescribed geometric parameters". IEEE Antennas and Propagation Magazine, Vol. 40, No. 2, April 1998

[6] Thomas Milligan, Modern Antenna Design,McGraw-Hill, 1985 ,pp.239-249.

[7] Per-Simon Kildal,Foundations of Antennas- A Unified Approach, Studentliterature,2000,p.10

[8] Paul Wade, Parablic Dish Antennas, N1BWT, 1998,chap. 4,pp.5-14.

[9] Jeffery M. Litchman,"Methods of determining the antenna focal point,"Radio Astronomy Supplies,Application note 8, October 2002

[10] C.A. Balanis, Antenna Theory, $3^{\text {rd }}$ ed,john Wiley and Sons,2005,pp. 34-35 